\title{
Vol. 68, No. SS-8
}

In the $M M W R$ Surveillance Summary "Population-Based Active Surveillance for Culture-Confirmed Candidemia Four Sites, United States, 2012-2016," on page 9, the second and third footnotes ( ${ }^{\dagger}$ and ${ }^{\S}$ ) for Table 3 should have read

${ }^{\dagger}$ Culture positive for Candida was obtained $<3$ days after admission for a patient with a recent health care exposure.

$\S$ Culture positive for Candida was obtained $<3$ days after admission for a patient without a recent health care exposure. 